Ann. Génét. Sél. anim., I969, 1 (I), 27-3I.

\title{
VERLÄNGERTE TRÄCHTIGKEIT BEIM PINZGAUER RIND
}

\author{
F. PIRCHNER
}

Institut jür Tierzucht und Haustiergenetik, Tierärztliche Hochschule, Wien, (Österreich)

\section{ZUSAMMENFASSUNG}

Eine Verlängerung der Trächtigkeit war durch Homozygotie eines recessiven Gens im Fötus bedingt. Die Föten waren zum Zeitpunkt der Geburt überschwer, mussten daher entweder durch Embryotomie oder durch forcierte Extraktion entwickelt werden. Die damit verbundene Schädigung des Muttertieres in Verbindung mit fehlender Vorbereitung des Euters führte in allen Fällen zur Schlachtung des Muttertieres. Das Gen wurde offensichtlich durch einen zugekauften Zuchtstier in die Herde gebracht. Zum Auftreten von Homozygoten aber kam es erst, als ein Enkel dieses Stieres in derselben Herbe verwendet worden war.

Die Dauer der Trächtigkeitsperiode beim Rind weist eine nicht unerhebliche Variabilität auf, die überdies relativ stark erblich beeinflusst ist (RENDEL, I959). Darüber hinaus aber kommen Trächtigkeitsverlängerungen vor, die pathologischen Charakter tragen. Solche Fälle sind von mehreren Rassen berichtet worden. Sie unterscheiden sich voneinander vielfach in klinischer Hinsicht. LAUVERGNF, I968, hat den Grossteil der in der Literatur mitgeteilten Fälle in einem Katalog aufgezählt und kurz beschrieben.

Die im folgenden berichteten fünf Fälle verlängerter Trächtigkeit sind in den Beständen einer Stierhaltungsgenossenschaft in der Umgebung von Hermagor. Kärnten, aufgetreten ( ${ }^{1}$ ). Die Trächtigkeitsperioden waren um I I/2 bis 3 Monate verlängert. In zwei Fällen wurde Embryotomie versucht, in einem dritten wurde das Kalb par force extrahiert. In den beiden anderen wurde die Kuh, nachdem die Schwierigkeiten durch die früheren Fälle bekanntgeworden waren, nach der Diagnose geschlachtet. Die Kühe zeigten fast keine Vorbereitung der Euter. Die Kälber lebten vor Beginn der Geburt, starben aber während, oder kurz nach,

(1) Eine kurze Mitteilung über diesen Erbfehler wurde bei der Tagung der Europäischen Vereinigung fïr Tierzucht, Oslo 1967 , gemacht. Catalogue Y,auvergne, $n^{\circ} 99$. 
der Geburt. Es kamen männliche und weibliche Kälber vor, wenn auch das genaue Geschlechtsverhältnis sich nicht mehr rekonstruieren lässt. Die Gewichte der Kälber waren weit über denen normaler Pinzgauerkälber. Ein gewogenes Kalb erreichte im ausgeschlachteten Zustand, also ohne Magen-Darmtrakt, ein Gewicht von $8 \mathrm{o} \mathrm{kg}$. Post-mortem-Untersuchungen wurden keine durchgeführt, doch wurde bei dem ausgeschlachteten Kalb eine auffallend dunkle Färbung der Nieren beobachtet. Die drei Kühe, von denen nur eine unter der offiziellen Milchkontrolle gestanden ist, gaben auch nach der Geburt bzw. Embryotomie der Kälber wenig Milch, sodass sie geschlachtet wurden. Bei den beiden anderen ist die Geburt nicht erst abgewartet worden, sondern sie wurden, wie schon erwähnt, nach Feststehen der Diagnose geschlachtet. Der durch diesen Erbfehler verursachte Schaden ist demnach für die betroffene Genossenschaft beträchtlich, da nicht nur die Kälber, sondern praktisch auch die Milchkïhe als verloren angesehen werden müssen.

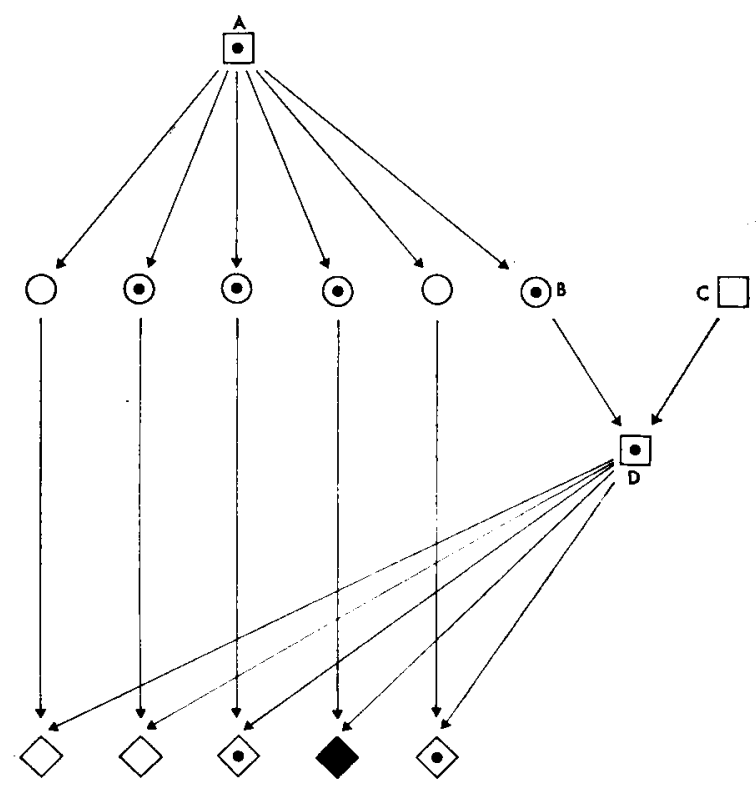

Aвв. 1. - Stammbaum eines Fötus mit verlängerter Trächtigkeit.

Fıg. r. - Pedigree d'un fatus ayant présenté une gestation prolongée.

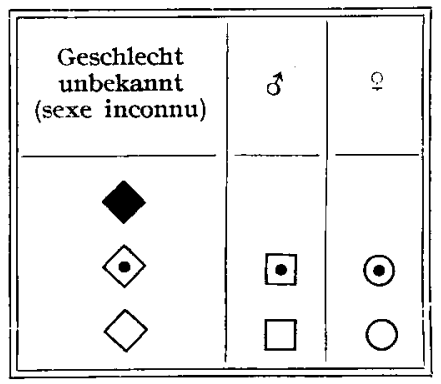

Fötus mit verlängerter Trächtigkeit (foctus à gestation prolongée).

Heterozygot (hétérozygote).

Homozygot normal (homozygote normal). 
Die beobachtete verlängerte Trächtigkeit ist offensichtlich durch Homozygotie eines rezessiven Gens verursacht. In der Stierhaltungsgenossenschaft wurde nur jeweils ein Stier verwendet, sodass alle Nachkommen eines Jahrgangs mit wenigen Ausnahmen (zugekaufte Kühe) von ihm abstammten. Nach seinem Abgang wurde einer seiner Enkel (D) als Deckstier eingestellt. Die Föten, die verlängerte Trächtigkeit verursacht haben, stammten zur Gänze von Stier $D$. Die Abstammungsverhältnisse sind in Abbildung I dargestellt. Offensichtlich war Stier A heterozygot für ein Gen n, das bei Homozygotie zu verlängerter Trächtigkeit führt. Etwa die Hälfte seiner Töchter musste daher das fehlerhafte Gen von A erhalten haben, was sich aber im heterozygoten Zustand klinisch nicht auswirken konnte. Eine dieser heterozygoten Töchter (B) aber wurde die Mutter des später verwendeten Deckstiers $D$, der väterlicherseits von einem Besamungsstier C abstammte. Bei Paarung von $\mathrm{D}$ mit den Töchtern von A, also mit seinen Halbtanten, muss es demnach in einem Achtel der Fälle zu Homozygotie und damit zu Verlängerung der 'Trächtigkeit kommen.

Die Stierhaltegenossenschaft umfasste etwa Ioo bis I20 faselbare weibliche Rinder. Der Stier D folgte unmittelbar auf Stier A, sodass, nachdem A ja durch fünf Jahre in Verwendung gestanden war, etwa die Hälfte der weiblichen Tiere, also etwa an die 50, Töchter von A waren. Der Stier D wurde aber nur während eines Zeitraums von 9-ro Monaten als Deckstier verwendet, sodass etwa um 40 Töchter des Stieres A von ihm gedeckt worden sein sollen, vorausgesetzt allerdings, dass die Sprünge mehr oder weniger gleichmässig über das ganze Jahr verteilt waren. Unter diesen Annahmen entspricht die Häufigkeit der Trächtigkeitsverlängerungen (5) den Erwartungen unter Annahme eines rezessiven Erbganges.

Zur Erleichterung eines Vergleiches sind die in der Literatur angeführten Fälle in Tabelle I zusammengefasst. Vor allem treten Unterschiede in bezug auf Gewicht und Beschaffenheit des Fötus auf. Die Verlängerung der Trächtigkeitsdauer ist dagegen so variabel, dass sich damit die berichteten Fälle nur schwer voneinander abgrenzen lassen. In Schwarzbunten war die um I-3 Monate verlängerte Trächtigkeit mit Übergewicht der Kälber verbunden, während in Schwedischem Rotvieh die Verlängerung stärker war (2-7 Monate), das Gewicht der Föten aber im Bereich der normalen Variabilität verblieben ist. Die von Guernseys berichteten Fälle zeigen sehr grosse Variabilität der Trächtigkeitsdauer, während Gewicht und Entwicklung der Kälber einem 7-Monate-Fötus entsprochen haben und dementsprechend auch Hypotrichose vorhanden war. Beim Finnischen Rind war ebenfalls Trächtigkeitsverlängerung mit Haarlosigkeit der Kälber verbunden, erstere aber relativ wenig variabel. Auch scheinen einige Kälber lebend geboren worden zu sein.

Die hier betichteten Fälle ähneln klinisch den von JASPER (I950) mitgeteilten. Hier wie dort war die Trächtigkeitsperiode um nicht mehr als 3 Monate verlängert und mit Übergewicht der Kälber verbunden. Nun existieren allerdings keine genealogischen Verbindungen zwischen Pinzgauern und Schwarzbunten, sodass es sich um verschiedene Mutationen entweder desselben oder eines anderen Locus mit ähnlicher Funktion handeln dürfte. Welche der beiden Hypothesen zutrifft, kann auf Grund der vorliegenden Information allerdings nicht entschieden werden. 


\section{TABELLE, I}

TABLEAU I

Erblich bedingte Trächtigkeitsverlängerung in Rindern (Gestation prolongée héréditaire chez les bovins)

\begin{tabular}{|c|c|c|c|c|c|}
\hline $\begin{array}{c}\text { Trächtigkeits- } \\
\text { dauer } \\
\text { Variations- } \\
\text { bereich in Tagen } \\
\text { (Intervalles } \\
\text { de variation } \\
\text { de la durée } \\
\text { de gestation } \\
\text { en jours) }\end{array}$ & $\begin{array}{c}\text { Gewicht und } \\
\text { Entwicklungs- } \\
\text { status } \\
\text { (Poids et état } \\
\text { d'évolution) }\end{array}$ & $\begin{array}{l}\text { Besonderheiten } \\
\text { (particularités) }\end{array}$ & $\begin{array}{l}\text { Rasse } \\
\text { (Races) }\end{array}$ & $\begin{array}{c}\text { Autor } \\
\text { (Auteurs) }\end{array}$ & $\begin{array}{c}\text { Katalog } \\
\text { Lauvergne }\end{array}$ \\
\hline $332-500$ & $\begin{array}{c}\text { normaler Varia- } \\
\text { tionsbereich }\end{array}$ & $\begin{array}{l}\text { Missbildg. d. In- } \\
\text { zisiven, Akro- } \\
\text { megalie }\end{array}$ & Schw. Rotvieh & HALIGREN & 92 \\
\hline $292-526$ & $\begin{array}{l}\text { entspr. 7-Mona- } \\
\text { te-Fötus }\end{array}$ & Hypotrichose & Guernseys & KENNEDY & 95 \\
\hline $290-363$ & $\begin{array}{l}\text { z. Tl. lebend ge- } \\
\text { boren }\end{array}$ & Hypotrichose & Finn. Rinder & MAIJALA & 93 \\
\hline $\begin{array}{l}290-370 \\
300-360\end{array}$ & $\begin{array}{l}\text { z. T1. lebend, } \\
\text { Uebergewicht } \\
\text { z. T1. lebend, } \\
\text { Uebergewicht }\end{array}$ & & $\begin{array}{l}\text { Holstein- } \\
\text { Friesen } \\
\text { Pinzgauer }\end{array}$ & $\begin{array}{l}\text { JASPER } \\
\text { Gegenw. Publ. }\end{array}$ & $\dot{-}$ \\
\hline
\end{tabular}

Es soll hier vielleicht kurz darauf verwiesen werden, da $\beta$ auf Grund einer Reihe von neueten Untersuchungen (PLUM, ANDERSEN and SWIGER, I965; EvERETT und MAGEE, I965) Trächtigkeitsdauer und Geburtsgewicht relativ eng miteinander korreliert sind, und zwar sowohl auf phänotypischer wie auch genetischer Basis. Weiterhin ist Geburtsgewicht mit späterem Gewicht beim Rind sowohl genetisch wie auch phänotypisch korreliert. In den letzten Jahren wurde der Körpergrösse beim Rind im Interesse der Fleischerzeugung vermehrte Beachtung geschenkt. Es ist daher nicht ausgeschlossen, dass Selektion auf Zuwachs und Körpergrösse zur Steigerung des Geburtsgewichtes und im weiteren Verlauf zur Verlängerung der Trächtigkeitsdauer führt und dass auf diese Weise Gene ausgelesen wurden, bei denen die Verlängerung der Trächtigkeitsdauer pathologischen Charakter hat. Dies würde allerdings implizieren, dasş das Gen auch in heterozygotem Zustand sich auf das Körpergewicht auswirkt.

Auf das Vorkommen verlängerter Trächtigkeit bin ich durch Dr. MARTIN. Hermagor, aufmerksam gemacht worden. Dr. MARTrN hat die klinischen Daten beigesteuert und war, ebenso wie Dr. KörBER, Klagenfurt, bei der Erhebung der Daten behilflich, wofür den Herren auch an dieser Stelle gedankt sei. 


\section{RÉSUMÉ}

Gestation prolongée en bétail de Pinzgau

Dans le bétail de Pinzgau, un gène récessif porté par le fotus est responsable de l'allongement de la gestation. La taille du foetus nécessite l'embryotomie ou l'extraction forcée. Le dommage causé à la mère joint à l'incomplète préparation de la mamelle a conduit, dans 5 cas, à l'abattage de la vache. Le gène a été introduit dans le troupeau par un taureau venu de l'extérieur. Les homozygotes sont apparus lorsqu'un petit-fils et des filles dudit taureau ont été croisés.

\section{SUMMARY}

\section{Eixtended Gestation in Pinzgau cows}

A prolongation of the gestation period in Pinzgau cattle was caused by homozygosity of the fœtus for a recessive gene. The fœetus was oversize so that embryotomy or forced extraction had to be employed. The damage thus caused to the dam, together with insufficient preparation of the udder, led in all five cases to the slaughter of the cows. The gene was introduced into the herd by a bull purchased from outside. The homozygotes appeared when a grandson of this bull was mated with daughters of the bull.

\section{LITERATUR}

Everett, R. W., and W. T. MAGEe, I965. Maternal ability and genetic ability of birth weight and gestation length. J. Dairy Sci., 48, 957-96r.

Haligren W., I95I. Abnormt läng dräktighet hos ko. Nord. Veter. Med., 3, ro43-106o.

JASPer D. E., r950. Prolonged gestation in the bovine. Cornell. Veter., 40, r65-172.

KENNEDY P. C., J. W. KENDRICK and C. STORMonT, 1957. Adenohypophyseal aplasia, an inherited defect associated with abnormal gestation in Guernsey cattle. Cornell. Veter., 47, 165-178.

I,AUVERgne J. J., I968. Catalogue des anomalies héréditaires des bovins (Bos taurus $\mathrm{L}_{\text {.) }}$. Bull. techn. Dep. Génét. anim., (I), I.N.R.A., nº 92-99.

MaIJALA K., V. VaINIKainen, and G. InNDSTRöm, 1964. On the relation between blood group genes and $\mathrm{a}$ lethal gene for hairlessness and prolonged gestation. Ann. Agr. Fenn., 3, 279-285.

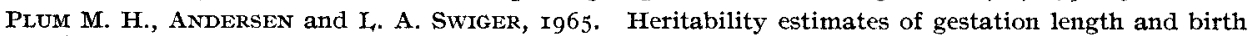
weight in Holstein-Friesian cattle and their use in selection indexes. J. Dairy Sci., 48, 1672-1675.

RENDEL J., 1959. Factors influencing gestation length in Swedish breeds of cattle. Z. Tierz. Zucht. Biol., 73, I $17-128$. 